\title{
Impact of Physicians' Personalities and Behavioral Traits on Treatment-Related Decision-making for Elderly Acute Myeloid Leukemia
}

\author{
Xia Wu, $M D^{7}$, Yi-nan Jiang, $M D^{2}$, Yue-lun Zhang, $P h D^{3}$, Jia Chen, $M D^{7}$, Yue-ying Mao, $M D^{7}$, \\ Lu Zhang, $M D^{7}$, Dao-bin Zhou, $M D^{7}$, Xin-xin Cao, $M D^{7}$, and Jian $L^{i}, M^{7}$
}

\begin{abstract}
'Department of Hematology, Peking Union Medical College Hospital, Peking Union Medical College and Chinese Academy of Medical Sciences, Beijing, People's Republic of China; ${ }^{2}$ Department of Psychology, Peking Union Medical College Hospital, Peking Union Medical College and Chinese Academy of Medical Sciences, Beijing, People's Republic of China; ${ }^{3}$ Medical Research Center, Peking Union Medical College Hospital, Peking Union Medical College and Chinese Academy of Medical Sciences, Beijing, People's Republic of China.
\end{abstract}

BACKGROUND: Elderly patients with acute myeloid leukemia (AML) can be treated with intensive therapy, lowintensity therapy, or best supportive care. Medical decision-making might be affected by physicians' occupational and non-occupational factors.

OBJECTIVE: To explore the impact of physicians' personalities and behavioral traits on treatment-related decision-making for elderly AML patients.

DESIGN: A nationwide cross-sectional survey.

PARTICIPANTS: Hematologists in mainland China $(N=$ 529; response rate $64.5 \%)$.

MAIN MEASURES: The medical decision-making for elderly AML patients was evaluated using 6 clinical vignettes. Hematologists' attitudes toward risk and uncertainty, Big Five personality traits, and decision-making styles were assessed using binary lottery choices and well-recognized self-report inventories.

KEY RESULTS: The resulting binary regression model in predicting treatment intensity contained professional title group $(\mathrm{OR}=0.012,95 \% \mathrm{CI} 0.001$ to $0.136, P<0.001)$, conscientiousness $(\mathrm{OR}=0.336,95 \% \mathrm{CI} 0.121$ to $0.932, P$ $=0.036)$, extraversion $(\mathrm{OR}=0.403,95 \% \mathrm{CI} 0.166$ to $0.974, P=0.044)$, conscientiousness by title group $(\mathrm{OR}=$ $2.009,95 \%$ CI 1.100 to $3.667, P=0.023)$, and extraversion by title group $(\mathrm{OR}=1.627,95 \% \mathrm{CI} 0.965$ to $2.743, P=$ 0.068) as predictors of therapy intensity preference. Junior physicians with a higher level of extraversion (mean difference $=0.27 ; 95 \%$ CI 0.07 to $0.45 ; P=0.009$ ) or conscientiousness (mean difference $=0.19 ; 95 \%$ CI 0.01 to $0.36 ; P=0.028$ ) tended to prescribe more intensive therapy. Meanwhile, no significant correlation was found between physicians' personalities or behavioral traits and treatment-related decision-making in senior physicians. CONCLUSIONS: Physicians' personalities contribute to treatment-related decision-making for elderly AML patients, depending on the professional titles. More

Xia Wu and Yi-nan Jiang contributed equally to this study.

Jian Li and Xin-xin Cao contributed equally as co-corresponding authors.

Prior Presentations This work was presented as a poster at the 62nd ASH Annual Meeting and Exposition (December 5, 2020).

Received August 18, 2020

Accepted December 13, 2020

Published online January 28, 2021 extravert or conscientious attending physicians tended to prescribe more intensive therapy. Meanwhile, the decisions made by chief and associate chief physicians were not impacted by their personal traits. Junior physicians should be aware of such potential influence when making medical decisions.

KEY WORDS: elderly acute myeloid leukemia; treatment-related decisionmaking; personality; physician

J Gen Intern Med 36(10):3023-30

DOI: $10.1007 / \mathrm{s} 11606-020-06467-\mathrm{w}$

(C) Society of General Internal Medicine 2021

\section{INTRODUCTION}

Acute myeloid leukemia (AML) is often observed in elderly patients, with a median age of 68 years at diagnosis, and the treatment outcomes for elderly AML patients remain poor. ${ }^{1}$ In general, regimens for elderly AML patients can be divided into standard intensive induction, low-intensity therapy, and best supportive care. ${ }^{2}$ Traditional intensive induction therapy has higher response rates but can also result in lethal complications, ${ }^{3}$ while low-intensity regimens with lower toxicity have unsatisfactory response rates. ${ }^{4}$ Thus, although algorithms and a consensus regarding treatment for elderly AML patients have been proposed, the treatment strategy is still individualized due to the heterogeneity of the disease biology and the paucity of strong evidence in clinical practice. ${ }^{5,6}$ Therefore, such medical decision-making can be affected by factors beyond medical knowledge, including factors associated with the patient, the physician, and the patient-physician relationship.

Several studies have expounded the complexities of medical decision-making. ${ }^{7,8}$ Previous studies have found that personality traits, uncertainty aversion, and behavioral characteristics such as decision-making styles may influence the medical decision-making process. ${ }^{8}, 9$ For instance, Poulton et al. found that in end-of-life decision-making, a judging personality was associated with increased treatment withdrawal and hastening of death. ${ }^{9}$ Saposnik et al. recognized the 
association between cognitive bias and diagnostic inaccuracies in more than one-third of the published cases. ${ }^{8}$ Furthermore, such non-clinical influences on medical decision-making may vary by geographical region and ethnicity. ${ }^{10,11} \mathrm{~A}$ study by Bories et al. focused on French physicians and found that the medical decisions made for elderly AML patients were affected by physicians' tolerance of uncertainty and by rationality in male physicians. ${ }^{12}$ It is of interest to investigate whether physicians' personal characteristics affect medical decisionmaking for elderly AML patients. Therefore, our study aimed to determine whether personalities and behavioral characteristics, including the risk and uncertainty aversion and decision-making style of hematologists, affect the medical decision-making for elderly AML patients in China.

\section{METHODS}

\section{Survey Design}

This study was approved by the Ethics Committee of Peking Union Medical College Hospital. All participants provided informed consent to participate in this study. This study was an anonymous cross-sectional survey of hematologists in mainland China. Specialized hematologists at diverse stages of their careers, including attending physicians, associate chief physicians, and chief physicians practicing in public hospitals in mainland China, were eligible. A pilot study was performed with 21 hematologists, and the data were analyzed to revise the questionnaire draft. The final version of the survey took approximately $15 \mathrm{~min}$ to complete. The survey link was sent to hematologists in mainland China on July 27, 2019, via the WeChat social media platform, which is widely used in China and had over one billion active users in the first quarters of 2018 and 2019. ${ }^{13}$ Before the participants began the survey, the goal of the study was introduced, and they were informed that the survey was anonymous, voluntary, and without compensation. Data were collected through a 2-week period until August 10, 2019.

\section{Questionnaire}

The questionnaire sent to the participants was in Chinese. The complete questionnaire is provided as supplementary material. It contained three parts. The first part was basic information regarding the hematologists' demographic and occupational characteristics: gender, age, educational background, subspecialty, occupational experience, selfevaluation of experience with AML patients, hospital level and size, and physicians' professional titles. In China, professional titles in medicine include attending physician, associate chief physician, and chief physician, which is similar to lecturer, assistant professor, associate professor, and professor in academic rank.

The second part contained six clinical vignettes (Table 1) for the purpose of investigating clinical decision-making for elderly patients with AML. The vignettes, which were derived from real clinical cases and revised by two experienced hematologists, represented cases of elderly AML that were difficult owing to disputable therapeutic choices. Each vignette exhibited a distinct and representative situation regarding age, comorbidities, performance status, functional reserves, and cytogenetic and molecular features. For each vignette, there were three choices: (1) standard dose-intensive chemotherapy with the classical $7+3$ regimen (daunorubicin $60-90 \mathrm{mg} / \mathrm{m}^{2}$ or idarubicin $12 \mathrm{mg} / \mathrm{m}^{2}$, days $1-3$, with cytarabine $100 \mathrm{mg} / \mathrm{m}^{2}$ q12h, days 1-7); (2) low-intensity chemotherapy, with a reduced-dose $7+3$ regimen, hypomethylating drugs (decitabine or azacitidine), or priming regimen (cytarabine,

\section{Table 1 Six Vignettes of Elderly Patients with AML}

Instructions: For each vignette, choose the one you would recommend most among the following three choices:

(1) standard-dose intensive chemotherapy, including the classical $7+3$ regimen (daunorubicin $60-90 \mathrm{mg} / \mathrm{m}^{2}$ or idarubicin $12 \mathrm{mg} / \mathrm{m}^{2}$, days $1-3$, with cytarabine $100 \mathrm{mg} / \mathrm{m}^{2} \mathrm{q} 12 \mathrm{~h}$, days $1-7$ );

(2) low-intensity chemotherapy, including a reduced-dose 7+3 regimen, hypomethylating drugs (decitabine or azacitidine), or CAG regimen (cytarabine, aclarubicin, G-CSF); or

(3) best supportive care (without chemotherapies).

Vignette 1: A 70-year-old woman with an 11-year history of hypertension controlled by ACEIs. Her LVEF was $59 \%$. ECOG 1 . CBC: WBC $11 \times 10^{9} / \mathrm{L}$, hemoglobin $100 \mathrm{~g} / \mathrm{L}$, platelets $78 \times 10^{9} / \mathrm{L}$, peripheral blast $12 \%$. BMA: FAB2 AML with favorable karyotype translocation $(8 ; 21)$.

Vignette 2: A 66-year-old male with a long history of smoking. He was diagnosed with coronary artery disease with coronary artery stenting in 2011 , after which he quit smoking. Recently, he lost $5 \%$ of his body weight (from 80 kilograms to 76 kilograms). His LVEF was 51\%. ECOG 2. CBC: WBC $82 \times 10^{9} / \mathrm{L}$, neutrophils $0.3 \times 10^{9} / \mathrm{L}$, hemoglobin $92 \mathrm{~g} / \mathrm{L}$, platelets $50 \times 10^{9} / \mathrm{L}$, peripheral blast $55 \%$. BMA: FAB2 AML with $66 \%$ blast cells, normal karyotype, and FLT3-ITD ${ }^{\text {low }}$ mutation.

Vignette 3: A 72-year-old male with no comorbidity. He had untreated myelodysplastic syndrome (IPSS 0.5) since 2011. He had worsening anemia since 2016 and was still afebrile and independent of transfusion. ECOG 2 . CBC: WBC $1 \times 10^{9} / \mathrm{L}$, neutrophils $0.3 \times 10^{9} / \mathrm{L}$, hemoglobin $90 \mathrm{~g} / \mathrm{L}$, platelets $120 \times 10^{9} / \mathrm{L}$, peripheral blast $6 \%$. BMA: FAB2 AML with $40 \%$ blast cells, and complex karyotype (inversion 3, 5q-, 7-).

Vignette 4: A 61-year-old male. He had asymptomatic coronary artery stenosis (70-90\%) with normal cardiac function. He felt feeble recently. ECOG 2. CBC: WBC $15 \times 10^{9} / \mathrm{L}$, hemoglobin $78 \mathrm{~g} / \mathrm{L}$, platelets $30 \times 10^{9} / \mathrm{L}$, peripheral blast $13 \%$. BMA: FAB2 AML with normal karyotype.

Vignette 5: A 63-year-old male with type 2 diabetes mellitus. He had small infarcts in the left cerebrum with no complications after successful thrombolysis 18 months ago. ECOG 2. CBC: WBC $55 \times 10^{9} / \mathrm{L}$, hemoglobin $78 \mathrm{~g} / \mathrm{L}$, platelets $30 \times 10^{9} / \mathrm{L}$, peripheral blast $25 \%$. BMA: FAB2 AML with pathological hematopoiesis of erythrocytes and megakaryocytes and complex karyotype.

Vignette 6: A 70-year-old male with asymptomatic coronary artery stenosis (70-90\%) with normal cardiac function. He felt feeble recently. ECOG 2. $\mathrm{CBC}$ : WBC $15 \times 10^{9} / \mathrm{L}$, hemoglobin $78 \mathrm{~g} / \mathrm{L}$, platelets $30 \times 10^{9} / \mathrm{L}$, peripheral blast $13 \%$. BMA: FAB2 AML with normal karyotype.

G-CSF, granulocyte-colony-stimulating factor; LVEF, left ventricular ejection fraction; ACEIs, angiotensin-converting enzyme inhibitors; ECOG, Eastern Cooperative Oncology Group performance status; CBC, complete blood count; WBC, white blood cell; BMA, bone marrow aspiration; FAB, French-American-British; AML, acute myeloid leukemia 
aclarubicin, G-CSF); and (3) best supportive care (without chemotherapies).

The third part was intended to assess the hematologists' risk and uncertainty aversion, personality traits, and decisionmaking style. It consisted of three self-report scales that had been validated in representative Chinese surveys. ${ }^{14-16}$ Personality traits were measured by the Big Five Inventory (BFI), which consisted of 44 items assessed on a 5-point Likert scale. ${ }^{15}$ According to John et al., ${ }^{17}$ personality traits can be depicted using five factors: openness to experience, conscientiousness, extraversion, agreeableness, and neuroticism. Decision-making style was measured by the Melbourne Decision Making Questionnaire (DMQ) ${ }^{16}$ and the General Decision Making Style Questionnaire (GDMS). ${ }^{14}$ The Melbourne DMQ contains 22 items measured on a 3-point scale and focuses mainly on different decision-coping patterns, including vigilance, hypervigilance, buck-passing, and procrastination. ${ }^{18}$ The GDMS contains 20 items measured on a 5-point Likert scale and identifies four decision-making styles: rational, dependent, avoidant, and spontaneous + intuitive. ${ }^{14}$

${ }^{19}$ To investigate risk and uncertainty aversion in hematologists, three questions used an 11-point Likert scale (from 0 to 10) to measure the extent of willingness to take risks regarding a general situation, money, and health. Meanwhile, two questions with binary lottery choices were designed based on the study by Bories et al. ${ }^{12}$

\section{Statistical Analysis}

The categorical variables are presented as numbers (percentage), and the quantitative data are presented as mean value ( \pm standard deviation). Similar to the statistical methods used by Bories et al., ${ }^{12}$ we initially used the Kmeans clustering to analyze the 6 clinical vignettes in order to define two groups of hematologists with homogeneous treatment preference patterns for elderly patients with AML. The group where more hematologists tended to choose intensive chemotherapy (IC) was defined as the "intensive treatment group" (IC group), while the other was defined as the "non-intensive treatment group" (nonIC group). Because the decisions in the vignettes were ordinal, a score for the vignettes was calculated by summing the answers of all the vignettes so that lower scores were associated with decisions to choose more intensive chemotherapy.

Initially, comparisons were made between the IC group and the non-IC group: The Student $t$ test or MannWhitney $U$ test was used for continuous variables; the chi-square test or Fisher exact test was used to compare the categorical variables between the two groups; and the Spearman rank correlation test was used for ordinal variables. Based on our experience, we divided participants into two groups according to their professional titles. One group was the "junior title group" (junior group), which included all the attending physicians; the other group was the "senior title group" (senior group), containing the associate chief physicians and chief physicians. Subgroup analyses were performed to identify differences between the IC and non-IC participants in each group. As we did not employ any correction for the inflation of the probability of type I error, results from subgroup analysis were only interpreted as explorative findings.

Interactions between the title group and personality and behavioral traits were tested using binary logistic analysis. Multiple binary logistic regression analyses were performed using a backward selection based on log-likelihood tests with an exclusion criterion of $P \geq 0.1$ to exploratively identify the independent predictors of decision-making for elderly AML patients after assessment of the collinearity between the predictor variables. The selection of the predictor variables initially included in the model was based on a combination of clinical experience with a threshold of $P=$ 0.2. Age and gender were adjusted. For all analyses, a twosided $P<0.05$ was considered statistically significant. All analyses were computed using SPSS statistics V.22.0 (SPSS Inc., Chicago, IL).

\section{RESULTS}

\section{Demographic and Occupational Results}

Of the 820 eligible hematologists contacted, a total of 529 completed the survey; the response rate was $64.5 \%$. The hematologists had a broad geographical representation; they worked in hospitals located in 150 different counties and districts, which were distributed in 29 provinces and municipalities. Their detailed demographic and occupational characteristics are listed in Table 2. In total, 354 (67\%) were women. The mean age of the participants was $41.0 \pm 7.4$ years (range, 27-64 years), noting that most physicians retire before or at the age of 60 in China. Most of the hematologists worked in public tertiary hospitals $(504,95 \%)$ and academic hospitals (390, 74\%), which mostly had 30 to 59 beds in the division of hematology $(228,43 \%)$. Attending physicians accounted for $40 \%$ of the participants $(n=211)$, followed by associate chief physicians $(185,35 \%)$ and chief physicians $(133,25 \%)$. In China, graduates with bachelor, master, and doctoral degrees in medicine are all eligible to obtain certification as a hematologist after completing post-graduate training. Most of our participants had a master of medicine degree $(238,45 \%), 157$ (30\%) had a doctor of medicine degree, and $128(24 \%)$ had a bachelor of medicine degree. Nearly half of the participants (51\%) had treated 10 to 29 patients with AML in the previous year, while over half $(54 \%)$ had treated fewer than 10 elderly patients with AML. Regarding subspecialty of hematology, $225(43 \%)$ specialized further in acute leukemia. The median level of self-evaluated experience with AML patients was 7.6 \pm 1.5 on an 11-point scale from 0 to 10 . 
Table 2 Demographic and Occupational Characteristics of the Hematologists Surveyed in China

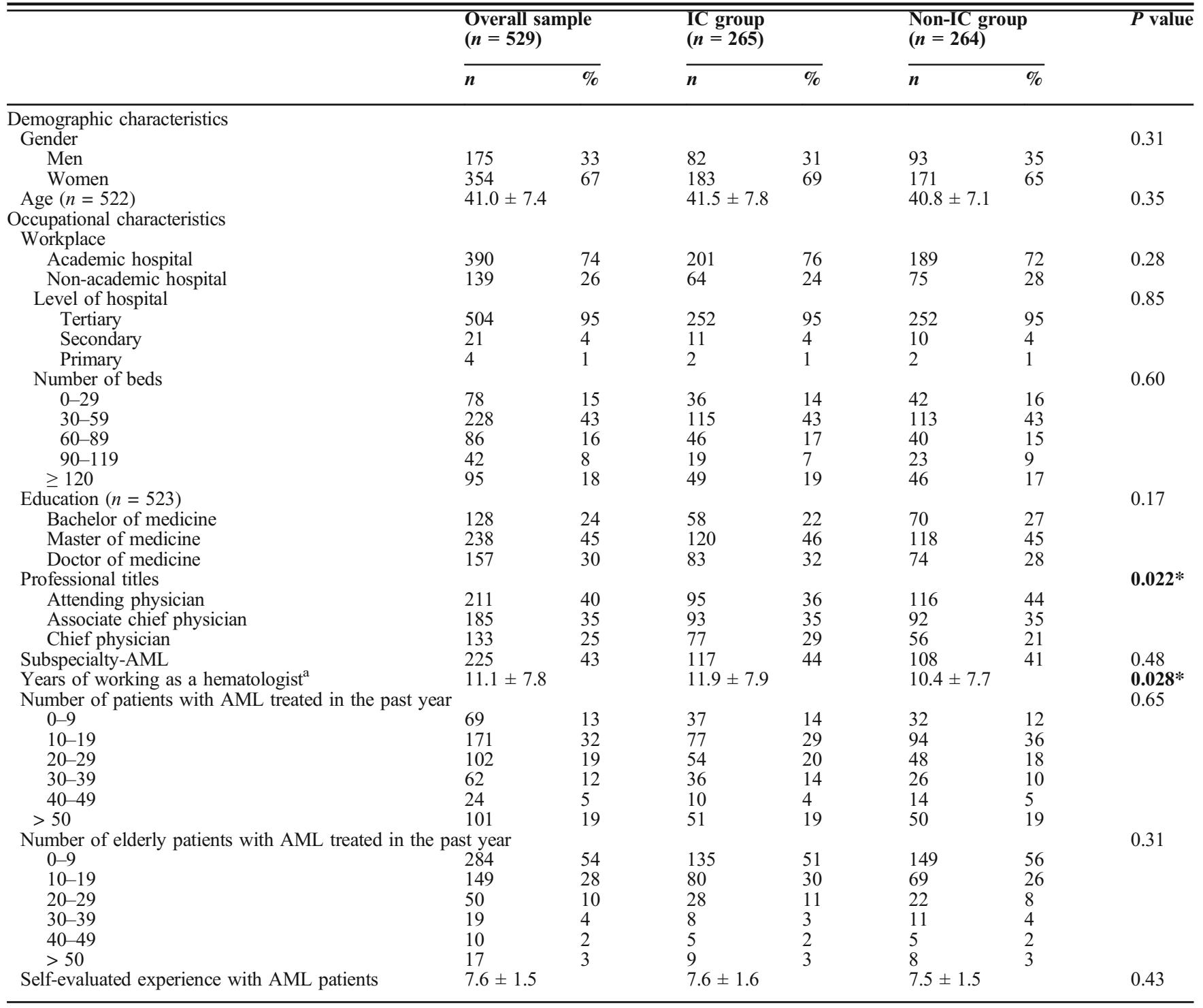

IC, intensive therapy; $A M L$, acute myeloid leukemia

${ }^{a}$ Years of working as a hematologist were counted after finishing residency

Bold emphasis and asterisk $(*)$ indicate statistical significance $(P<0.05)$

\section{Comparisons Between IC and Non-IC Groups}

The participants were classified into two groups via the $\mathrm{K}$-means cluster analysis based on the six vignettes. The IC group contained 265 hematologists, with $69 \%$ women $(n=183)$; the mean age was $41.5 \pm 7.8$ years. The nonIC group contained 264 hematologists, with $48 \%$ women $(n=171)$; the mean age was $40.8 \pm 7.1$ years. The mean vignette scores were $9.7 \pm 1.2$ in the IC group and $12.2 \pm$ 1.2 in the non-IC group. A lower score represents a more intensive choice. The distribution of the three choices in each vignette is presented in Figure 1. Compared with the hematologists in the non-IC group, those in the IC group had higher-level professional titles $(P=0.022)$ and more years of working experience $(11.9 \pm 7.9$ vs. $10.4 \pm$ 7.7, $P=0.028)$. No significant differences were found between the two groups concerning other occupational or non-occupational factors; attitudes toward risks or uncertainty; or any factors in the BFI, Melbourne DMQ, or GDMS (Table 3).

\section{Subgroup and Interaction Analyses}

Exploratory subgroup analysis was carried out in the junior ( $n$ $=211)$ and senior groups $(n=318)$. In the junior group, there were 95 participants classified in the IC group and 116 in the non-IC group. The participants in the IC group had a higher level of education $(P=0.023)$ and a higher ratio of working in academic hospitals than those in the non-IC group (75.8\% vs. $61.2 \%, P=0.024)$. For personality traits, the participants in the IC group had higher scores in extraversion (3.16 vs. 2.90 , $P=0.009$ ), conscientiousness ( 3.73 vs. $3.55, P=0.028$ ), and openness to experience (3.28 vs. $3.04, P=0.010)$. For the 

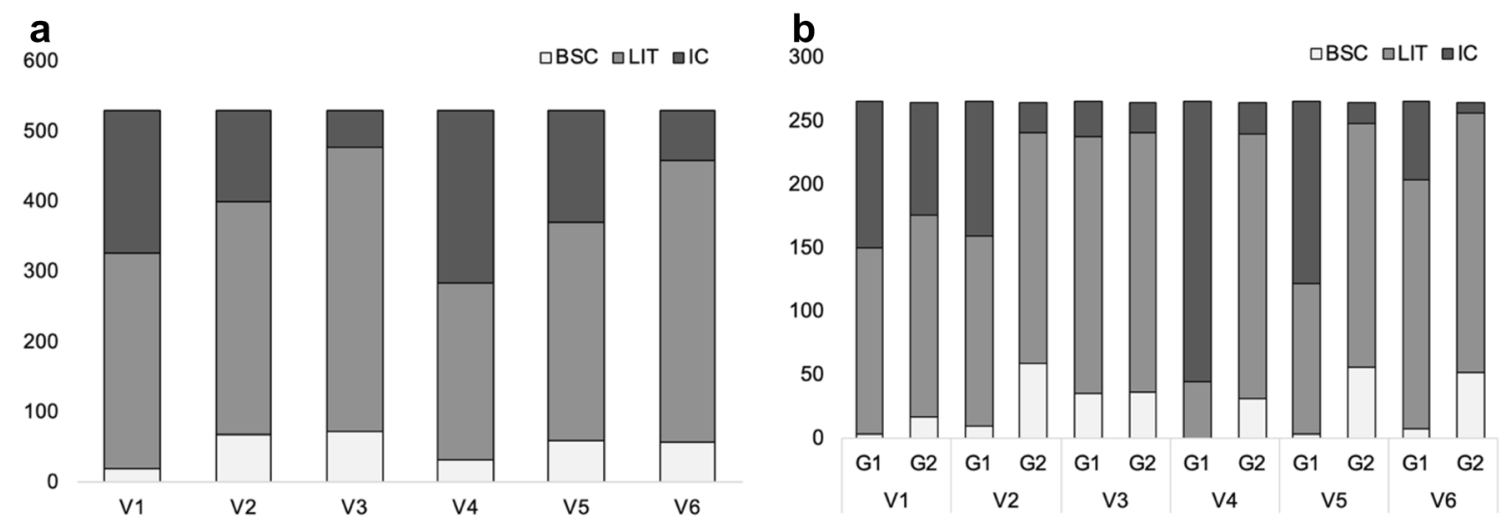

Figure 1 a Decision-making results for the six vignettes. b Decision-making results for each group. G1 represents the group with a preference for intensive therapy. G2 represents the group with a preference for non-intensive therapy. V1 to V6 represent vignette 1 to vignette 6 . IC, standard-dose chemotherapy; LIT, low-intensity chemotherapy; BSC, best supportive care.

decision-making style, the junior hematologists in the IC group had lower scores for avoidance on the GDMS $(2.35$ vs. $2.63, P=0.012)$. In the senior group, 170 participants were classified in the IC group and 148 in the non-IC group. Compared with the participants in the non-IC group, those in the IC group tended to have lower scores for conscientiousness (3.86 vs. 3.99, $P=0.060$ ) and higher scores for neuroticism (2.78 vs. $2.63, P=0.060)$, but the differences had no statistical significance. Interaction analyses were performed between the title group and the non-occupational characteristics, including uncertainty and risk aversion, personality traits, and decision-making characteristics (Table 4). Among the factors studied, significant interactions were found between title group and extraversion (junior vs. senior, 0.27 [0.07 to 0.45$]$ vs. $-0.08[-0.24$ to 0.08$], P=0.009$ ), conscientiousness $(0.19$ [0.01 to 0.36$]$ vs. $-0.13[-0.27$ to $0.01], P=0.005)$, neuroticism ( $-0.18[-0.38$ to 0.01$]$ vs.
$0.15[-0.01$ to 0.31$], P=0.009)$, openness $(0.24[0.06$ to $0.42]$ vs. -0.08 [ -0.22 to 0.07$], P=0.009)$ in the $\mathrm{BFI}$, and title group and avoidance $(-0.28[-0.53$ to 0.04$]$ vs. $0.06[-$ 0.12 to 0.24$], P=0.031$ ) in the GDMS.

\section{Analyses of Predictor Variables of Decision- making}

Fourteen variables were initially entered into the multiple binary logistic regression analysis: age, gender, educational background, years of working as a hematologist, title group, procrastination, extraversion, conscientiousness, openness, avoidance, extraversion by title group, conscientiousness by title group, openness by title group, and avoidance by title group. Ultimately, five predictor variables were kept in the final regression model, including title group $(\mathrm{OR}=0.012 ; 95 \% \mathrm{CI}$, 0.001 to $0.136 ; P<0.001)$, extraversion $(\mathrm{OR}=0.403 ; 95 \% \mathrm{CI}$, 0.166 to $0.974 ; P=0.044)$, and conscientiousness $(\mathrm{OR}=0.336$;

Table 3 Non-occupational Characteristics of Hematologists in China

\begin{tabular}{|c|c|c|c|c|}
\hline & Overall sample & TC group & Non-IC group & Pvalue \\
\hline Attitude toward uncertainty & $299.4 \pm 137.6$ & $303.9 \pm 142.8$ & $294.8 \pm 132.3$ & 0.45 \\
\hline Attitude toward risks & $334.7 \pm 120.4$ & $339.1 \pm 124.7$ & $330.3 \pm 116.0$ & 0.40 \\
\hline General attitude toward risks & $5.5 \pm 2.2$ & $5.6 \pm 2.3$ & $5.4 \pm 2.2$ & 0.27 \\
\hline Risks regarding money & $4.5 \pm 2.4$ & $4.5 \pm 2.4$ & $4.5 \pm 2.4$ & 0.98 \\
\hline Risks regarding health & $4.0 \pm 2.7$ & $4.0 \pm 2.6$ & $4.0 \pm 2.8$ & 0.87 \\
\hline \multicolumn{5}{|l|}{ Big Five Inventory } \\
\hline Extraversion & $3.02 \pm 0.72$ & $3.05 \pm 0.75$ & $2.99 \pm 0.68$ & 0.37 \\
\hline Agreeableness & $4.05 \pm 0.52$ & $4.04 \pm 0.55$ & $4.06 \pm 0.49$ & 0.55 \\
\hline Conscientiousness & $3.80 \pm 0.64$ & $3.81 \pm 0.66$ & $3.79 \pm 0.63$ & 0.74 \\
\hline Neuroticism & $2.78 \pm 0.72$ & $2.78 \pm 0.71$ & $2.78 \pm 0.72$ & 0.95 \\
\hline Openness & $3.25 \pm 0.67$ & $3.28 \pm 0.68$ & $3.22 \pm 0.67$ & 0.28 \\
\hline \multicolumn{5}{|c|}{ Melbourne Decision Making Questionnaire } \\
\hline Vigilance & $10.10 \pm 1.98$ & $10.13 \pm 1.99$ & $10.08 \pm 1.96$ & 0.74 \\
\hline Buck-passing & $2.90 \pm 2.66$ & $2.77 \pm 2.57$ & $3.04 \pm 2.74$ & 0.23 \\
\hline Procrastination & $2.59 \pm 2.26$ & $2.44 \pm 2.20$ & $2.75 \pm 2.31$ & 0.11 \\
\hline Hypervigilance & $4.69 \pm 2.80$ & $4.74 \pm 2.90$ & $4.64 \pm 2.70$ & 0.68 \\
\hline \multicolumn{5}{|c|}{ General Decision-Making Style Questionnaire } \\
\hline Rational & $4.10 \pm 0.59$ & $4.12 \pm 0.58$ & $4.08 \pm 0.60$ & 0.41 \\
\hline Dependent & $3.47 \pm 0.65$ & $3.46 \pm 0.65$ & $3.48 \pm 0.64$ & 0.68 \\
\hline Avoidant & $2.40 \pm 0.87$ & $2.35 \pm 0.86$ & $2.44 \pm 0.87$ & 0.24 \\
\hline Spontaneous + intuitive & $3.20 \pm 0.53$ & $3.18 \pm 0.55$ & $3.22 \pm 0.51$ & 0.41 \\
\hline
\end{tabular}


Table 4 Subgroup and Interaction Analyses of Non-occupational Characteristics Among Senior and Junior Groups

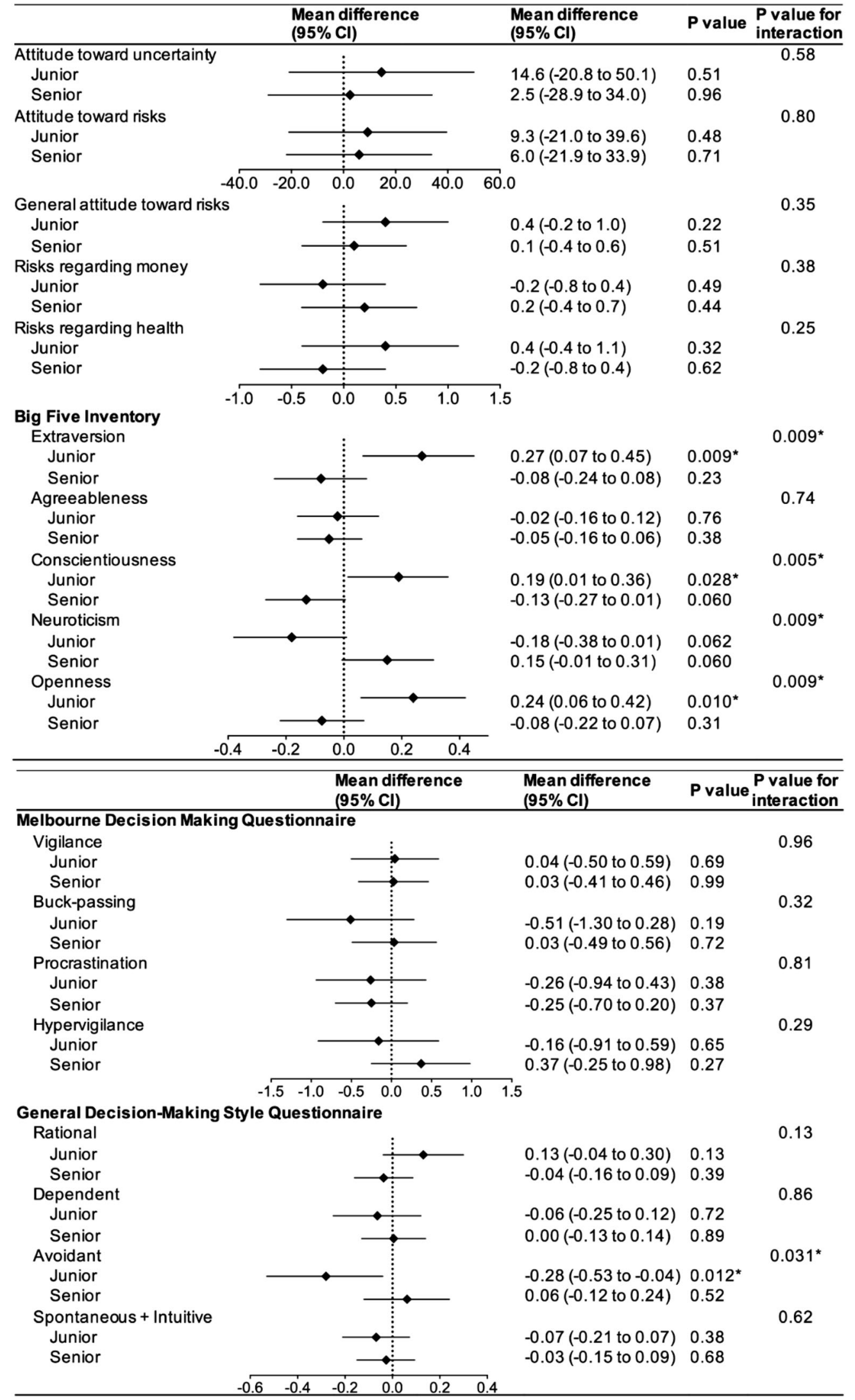

Junior refers to the junior group, which included all the attending physicians; senior refers to the senior group, which included all the associate chief physicians and chief physicians. The black dots represent mean differences between the IC and non-IC groups in each junior and senior group 
Table 5 Characteristics Associated with Belonging to the Non-IC group (Results from Logistical Regression Analysis)

\begin{tabular}{|c|c|c|c|}
\hline & $\overline{\mathrm{OR}}$ & $95 \% \mathrm{CI}$ & $P$ value \\
\hline Title group & 0.012 & 0.001 to 0.136 & $<0.001$ \\
\hline Extraversion & 0.403 & 0.166 to 0.974 & 0.044 \\
\hline Conscientiousness & 0.336 & 0.121 to 0.932 & 0.036 \\
\hline Interaction term $=$ conscientiousness $*$ title group & 2.009 & 1.100 to 3.667 & 0.023 \\
\hline Interaction term $=$ extraversion $*$ title group & 1.627 & 0.965 to 2.743 & 0.068 \\
\hline
\end{tabular}

95\% CI, 0.121 to $0.932 ; P=0.036$ ) (Table 5). The interaction term of conscientiousness by title group remained significant, indicating a significant difference in conscientiousness between the junior and senior groups in predicting the treatment intensity preference $(\mathrm{OR}=2.009 ; 95 \% \mathrm{CI}, 1.100$ to $3.667 ; P=0.023)$. For the junior hematologists, the IC group had a significantly higher level of conscientiousness than the non-IC group (mean difference $=0.19 ; 95 \% \mathrm{CI}, 0.01$ to $0.36 ; P=0.028$ ), while for the senior hematologists, the difference in conscientiousness between the IC and non-IC groups was not statistically significant (mean difference $=-0.13 ; 95 \% \mathrm{CI},-0.27$ to $0.01 ; P=$ 0.060 ). Another interaction term that was kept in the regression model was extraversion by the title group $(\mathrm{OR}=1.627 ; 95 \% \mathrm{CI}$, 0.965 to $2.743 ; P=0.068$ ). For the junior hematologists, the IC group had a significantly higher level of extraversion than the non-IC group (mean difference $=0.27 ; 95 \% \mathrm{CI}, 0.07$ to $0.45 ; P$ $=0.009)$, while for the senior hematologists, the difference in extraversion between the IC and non-IC groups was not statistically significant (mean difference $=-0.08 ; 95 \% \mathrm{CI},-0.24$ to $0.08 ; P=0.23$ ). As stated in the methodology, we cannot totally exclude the probability of false positive results due to the multiple tests conducted and the limited sample size.

\section{DISCUSSION}

In this cross-national study, we evaluated the relationship between physicians' personalities and behavioral characteristics and clinical decision-making for elderly AML patients. Our findings revealed that the predictive factors of treatment intensity preference differed based on physicians' professional titles.

Previous studies have addressed the effect of risk and uncertainty aversion and other personality traits, such as confidence, on physicians' decision-making preferences. ${ }^{20-23}$ In the study by Bories et al., factors in French physicians that affected their decision-making for elderly AML patients included aversion to uncertainty, number of AML patients, and expected utility among men. ${ }^{12}$ In contrast to their findings, neither risk aversion nor uncertainty aversion affected the medical decision-making in our study. One explanation is that personal traits may vary across cultures. Studies revealed that French have a relative high score in uncertainty avoidance, ${ }^{21}$, 24,25 while in our study, the professional title has an effect modification on the decision-making.

For attending physicians, personalities affect the decisionmaking. More extravert or conscientious attending physicians tended to prescribe more intensive therapy. In contrast to introversion, extraversion implies an energetic approach toward social situations and a tendency toward assertiveness and positive emotionality. ${ }^{26}$ Extraverts tend to be enthusiastic and assertive and to seek leadership positions. ${ }^{26}$ Oehler et al. also found that more extraverted individuals could tolerate higher risks. ${ }^{27}$ Consequently, it seems reasonable that attending physicians with higher extraversion scores are likely to choose more intensive therapies. Conscientiousness refers to the control of socially prescribed impulses and results in a tendency to display selfdiscipline and act dutifully. ${ }^{28}$ Senses of social responsibility and self-discipline may become intrinsic driving forces for conscientious attending physicians to prescribe more intensive therapy.

For associate chief physicians and chief physicians, it is interesting that no significant predictive factors concerning risk and uncertainty aversion, personality traits, or decisionmaking characteristics were found in relation to the therapy intensity preference. One possible reason may be that senior physicians generally have more clinical experience than junior attending physicians, so their medical decisions tend to be based more on clinical experience and are less likely to be influenced by personal characteristics.

Overall, this study demonstrated that medical decisions made by attending physicians, but not associate chief and chief attendings, could be impacted by their personalities. Junior hematologists should be aware of such potential influence when making medical decisions. This is especially important when the decision-making is highly value-laden and contentious, as in the management of elderly patients with AML. Junior hematologists should remind themselves to respect and focus more on individual patient's preferences, needs, and values.

Our study has several limitations. The study population might not fully represent all hematologists working in China. It would be better to use stratified sampling, although our convenience sampling method is more achievable. Besides, new targeted therapies such as venetoclax and enasidenib were not included in our study, while these drugs may change practice patterns of elderly AML in China in the future. In addition, although studies have demonstrated the congruence between physicians' responses to vignettes and their real choices made in the clinic, ${ }^{29,30}$ our methods of using vignettes for case delivery still limit the real-world validity of this study. Previously, some studies on patients have demonstrated the impact of physicians' personal traits on real clinical outcomes in other conditions. ${ }^{9,} 31,32$ In the future, researches on the actual treatment-related decisions made by hematologists are needed to further verify our findings. 
In conclusion, our study suggests that physicians' personalities contribute to medical decision-making for elderly AML patients, depending on the professional titles. Attending physicians with a higher level of extraversion or conscientiousness tended to prescribe more intensive therapy. Meanwhile, no correlation between physicians' personalities or behavioral traits and medical decision-making was observed in chief and associate chief physicians. Junior physicians should be aware of such potential influence when making medical decisions.

Supplementary Information The online version contains supplementary material available at https://doi.org/10.1007/s11606-02006467-w.

Acknowledgments: The authors thank all the hematologists who participated in the survey.

Corresponding Author: Xin-xinJian CaoLi, MD; Department of Hematology, Peking Union Medical College Hospital, Peking Union Medical College and Chinese Academy of Medical Sciences, Beijing, People's Republic of China (e-mail: caoxinxin@126.com).

Funding Institutional research funding was provided by the Nonprofit Central Research Institute Fund of Chinese Academy of Medical Sciences (Grant No. 2019-RC-HL-001, for X.-x.C.), the National Natural Science Foundation of China (Grant No. 81570195, for L.J.), and Beijing Natural Science Foundation (Grant No. 7182128, for L.J.).

\section{Compliance with Ethical Standards:}

Conflict of Interest: The authors declare that they do not have a conflict of interest.

Disclaimer: The funders had no role in study design, data collection and analysis, decision to publish, or preparation of the manuscript.

\section{REFERENCES}

1. Institute SEaERPotNC. SEER cancer stat facts: acute myeloid leukemia (AML) 2018. Available from: https://seer.cancer.gov/statfacts/html/amyl.html. Accessed 10 Sept 2019

2. Kantarjian HM, Thomas XG, Dmoszynska A, et al. Multicenter, randomized, open-label, phase III trial of decitabine versus patient choice, with physician advice, of either supportive care or low-dose cytarabine for the treatment of older patients with newly diagnosed acute myeloid leukemia. J Clin Oncol 2012;30(21):2670.

3. Podoltsev NA, Stahl M, Zeidan AM, et al. Selecting initial treatment of acute myeloid leukaemia in older adults. Blood Rev 2017;31(2):43-62.

4. Dombret $\mathbf{H}$, Seymour JF, Butrym A, et al. International phase 3 study of azacitidine vs conventional care regimens in older patients with newly diagnosed AML with>30\% blasts. Blood. 2015;126(3):291-299.

5. Rao AV. Fitness in the elderly: how to make decisions regarding acute myeloid leukemia induction. ASH Education Program Book 2016;2016(1):339-347.

6. Loberiza Jr FR, Cannon AC, Cannon AJ, et al. Insights on practice variations in the management of lymphoma and leukemia. Leuk Lymphoma 2014;55(11):2449-2456.

7. Hajjaj FM, Salek MS, Basra MK, et al. Non-clinical influences on clinical decision-making: a major challenge to evidence-based practice. J R Soc Med 2010;103(5):178-187.

8. Saposnik G, Redelmeier D, Ruff CC, et al. Cognitive biases associated with medical decisions: a systematic review. BMC Med Inform Decis Mak 2016;16(1): 138 .

9. Poulton B, Ridley S, Mackenzie-Ross $\mathbf{R}$, et al. Variation in end-of-life decision making between critical care consultants. Anaesthesia. 2005;60(11):1101-1105.
10. Malhotra J, Rotter D, Tsui J, et al. Impact of patient-provider race, ethnicity, and gender concordance on cancer screening: findings from Medical Expenditure Panel Survey. Cancer Epidemiol Prev Biomarkers 2017;26(12):1804-1811.

11. Cutler D, Skinner JS, Stern AD, et al. Physician beliefs and patient preferences: a new look at regional variation in health care spending. Am Econ J Econ Pol 2019;11(1):192-221.

12. Bories $\mathbf{P}$, Lamy $\mathbf{S}$, Simand $\mathbf{C}$, et al. Physician uncertainty aversion impacts medical decision making for older patients with acute myeloid leukemia: results of a national survey. Haematologica. 2018;103(12):2040-2048.

13. Tencent. Tencent Announces 2019 First Quarter Results 2019. Available from: https://www.tencent.com/en-us/articles/8003551557911908. pdf. Accessed 15 Sept 2019.

14. Dong JH. The influential factor and construction of factor model of risk decision-making. MS thesis, Northwest Normal University. 2006, 57 pages.

15. Schmitt DP, Allik J, McCrae RR, et al. The geographic distribution of Big Five personality traits: Patterns and profiles of human self-description across 56 nations. J Cross-Cult Psychol 2007;38(2):173-212.

16. Huang $\mathbf{R}$. The relationship between power and decision-making style. MS thesis, Hunan Normal University. 2016, 40 pages.

17. John OP, Naumann LP, Soto CJ. Paradigm shift to the integrative big five trait taxonomy. Handbook of personality: Theory and research 2008;3(2):114-158.

18. Mann L, Burnett P, Radford M, et al. The Melbourne Decision Making Questionnaire: An instrument for measuring patterns for coping with decisional conflict. J Behav Decis Mak 1997;10(1):1-19.

19. Spicer DP, Sadler-Smith E. An examination of the general decision making style questionnaire in two UK samples. J Manag Psychol 2005;20(2): 137-149.

20. Meyer AN, Payne VL, Meeks DW, et al. Physicians' diagnostic accuracy, confidence, and resource requests: a vignette study. JAMA Intern Med 2013;173(21):1952-1958.

21. Michel-Lepage A, Ventelou B, Nebout A, et al. Cross-sectional survey: risk-averse French GPs use more rapid-antigen diagnostic tests in tonsillitis in children. BMJ Open 2013;3(10):e003540.

22. Massin S, Ventelou B, Nebout A, et al. Cross-sectional survey: riskaverse French general practitioners are more favorable toward influenza vaccination. Vaccine. 2015;33(5):610-614.

23. Massin S, Nebout A, Ventelou B. Predicting medical practices using various risk attitude measures. Eur J Health Econ 2018;19(6):843-860.

24. Deschepper R, Grigoryan L, Lundborg CS, et al. Are cultural dimensions relevant for explaining cross-national differences in antibiotic use in Europe? BMC Health Serv Res 2008;8(1):123.

25. Borg MA. Prolonged perioperative surgical prophylaxis within European hospitals: an exercise in uncertainty avoidance? J Antimicrob Chemother 2013;69(4):1142-1144.

26. Wilt J, Revelle W. Extraversion. The Oxford handbook of the Five Factor Model. Oxford library of psychology. New York: Oxford University Press; 2017. p. 57-81.

27. Oehler A, Wedlich F. The relationship of extraversion and neuroticism with risk attitude, risk perception, and return expectations. J Neurosci Psychol Econ 2018;11(2):63-92.

28. Anderson C, Spataro SE, Flynn FJ. Personality and organizational culture as determinants of influence. J Appl Psychol 2008;93(3):702.

29. Peabody JW, Luck J, Glassman P, et al. Comparison of vignettes, standardized patients, and chart abstraction: a prospective validation study of 3 methods for measuring quality. Jama. 2000;283(13):17151722.

30. Peabody Jw, Luck J, Glassman P, et al. Measuring the quality of physician practice by using clinical vignettes: a prospective validation study. Ann Intern Med 2004;141(10):771-780.

31. Hojat M, Louis DZ, Markham FW, et al. Physicians' empathy and clinical outcomes for diabetic patients. Acad Med 2011;86(3):359-364.

32. Buining EM, Kooijman MK, Swinkels IC, et al. Exploring physiotherapists' personality traits that may influence treatment outcome in patients with chronic diseases: a cohort study. BMC Health Serv Res 2015;15(1):558-569.

Publisher's Note Springer Nature remains neutral with regard to jurisdictional claims in published maps and institutional affiliations. 